\title{
Perspectiva de género en Ciencias de la Salud: Igualdad y Terapia Ocupacional
}

\author{
Canosa Domínguez, Nereida María'; Groba González, Betania²; Nieto-Riveiro, Laura³ \\ Pousada García, Thais ${ }^{4}$ \\ ${ }^{1}$ Grupo TALIONIS-CITIC, Facultade de Ciencias da Saúde, \\ Universidade da Coruña, 0000-0001-8527-9845 \\ ${ }^{2}$ Grupo TALIONIS-CITIC, Facultade de Ciencias da Saúde, \\ Universidade da Coruña, 0000-0001-5547-2337 \\ ${ }^{3}$ Grupo TALIONIS-CITIC, Facultade de Ciencias da Saúde, \\ Universidade da Coruña, 0000-0003-3460-5895 \\ ${ }^{4}$ Grupo TALIONIS-CITIC, Facultade de Ciencias da Saúde, \\ Universidade da Coruña, 0000-0003-3356-0429
}

\section{RESUMEN}

La perspectiva de género hace referencia a un posicionamiento crítico de normatividad patriarcal que sustenta el sistema social en el que el género es un principio de jerarquización de espacios, recursos y valores. La Universidad como Institución transmisora de valores y promotora del cambio social debe educar en Igualdad. Desde la Facultad de Ciencias de la Salud, específicamente desde el Grado en Terapia Ocupacional, se aborda el género, como determinante social de la salud, y la perspectiva de género, de forma concreta y/o transversal, en el currículo. En este sentido en el curso 2012-2013 se implanta en la materia Terapia Ocupacional, Diversidad Funcional y Calidad de Vida, un módulo sobre Igualdad, Género, Diversidad y Salud destinado a alumnado de cuarto curso de la Titulación. En la programación se desarrollaron actividades de formación y sensibilización, analizando diversos contextos generadores de exclusión y que atentan contra los Derechos Humanos. Los principales resultados se tradujeron en un incremento de Trabajos Fin de Grado vinculados con las temáticas tratadas. Asimismo, las investigaciones iniciadas en el Grado impactaron en diversas esferas de 
educación y responsabilidad social. Es preciso reflexionar sobre el papel de la Universidad como Institución promotora de una ciudadanía más tolerante e igualitaria.

PALABRAS CLAVE: Terapia Ocupacional, Igualdad, Género, Salud, Derechos Humanos.

Canosa Domínguez, Nereida María; Groba González, Betania; Nieto-Riveiro, Laura, Pousada García, Thais (2020): Perspectiva de género en Ciencias de la Salud: Igualdad y Terapia Ocupacional. En De la Torre Fernández, E. (ed.) (2020). Contextos universitarios transformadores: Boas prácticas no marco dos GID. IV Xornadas de Innovación Docente. Cufie. Universidade da Coruña. A Coruña (págs. 295-304). DOI capítulo: https://doi.org/10.17979/spudc.9788497497756.295 DOI libro: https://doi.org/10.17979/spudc.9788497497756

\section{ABSTRACT}

The gender perspective refers to a critical positioning of patriarchal norms that support the social system in which gender is a principle of hierarchization of spaces, resources and values. The University as an institution that transmits values and promotes social change must educate on equality. From the Faculty of Health Sciences, specifically from the Degree in Occupational Therapy, gender is addressed as a social determinant of health, and the gender perspective, in a concrete and/or transversal way, in the curriculum. In this sense, in the 2012-2013 academic year, a module on Equality, Gender, Diversity and Health was implemented in the subject of Occupational Therapy, Functional Diversity and Quality of Life, aimed at students in the fourth year of the Degree. In the programming, training and awareness-raising activities were developed, analysing various contexts that generate exclusion and that threaten human rights. The main results were translated into an increase of Final Works linked to the topics covered. Likewise, the research initiated in the Degree had an impact on various areas of education and social responsibility. It is necessary to reflect on the role of the University as an institution promoting a more tolerant and egalitarian citizenship.

KEY WORDS: Occupational Therapy, Equality, Gender, Health, Human Rights. 


\section{INTRODUCCIÓN}

Nuestra sociedad se sustenta en un sistema patriarcal basado en relaciones jerárquicas y de poder de los hombres frente a las mujeres (Amorós, 1995; Cagigas Arriazu, 2000; Facio \& Fries, 2005). Según la Comisión Europea (2016) esa organización patriarcal repercute en la construcción del género atribuyendo "modelos de comportamiento para la feminidad y para la masculinidad". En ese sentido, el género es una identidad socialmente construida, al igual que la clase social, la raza, la sexualidad o la diversidad funcional, que puede generar situaciones de desigualdad. Esas desigualdades de género repercuten, según la Organización Mundial de la Salud (OMS, 2009), en la salud y en la justicia social de mujeres y niñas de todo el mundo. La Universidad como Institución transmisora de valores y promotora del cambio social debe comprometerse y educar en Igualdad.

A lo largo de este capítulo se pretende reflexionar sobre la importancia de incorporar la perspectiva de género en el ámbito universitario.

\section{Incorporación de la Perspectiva de género en la Docencia Universitaria}

La perspectiva de género hace referencia a un posicionamiento crítico de la normatividad patriarcal ya que centra su mirada en las necesidades, comportamientos y actitudes de la población, alejándose de un modelo androcéntrico que considera al hombre como sujeto universal. Así, una docencia con perspectiva de género es una docencia para las personas, teniendo en consideración sus características biológicas, pero también sus contextos sociales y culturales (Scott, 1986; Beckwith, 2005).

La Agencia para la Calidad del Sistema Universitario Catalán (AQU, 2019) destaca que "incorporar la perspectiva de género a la docencia mejora la calidad docente y la relevancia social de los conocimientos". Además, la Unión Europea concibe el género no sólo como una cuestión de justicia social, sino que afecta al propio rendimiento de la docencia y la investigación. 
En España, desde el año 2003 se han desarrollado distintas políticas que promueven la incorporación de esta mirada en el currículo universitario, de forma específica o transversal en las distintas Titulaciones (Alonso y Lombardo, 2016). Así, la Ley Orgánica 1/2004, de 28 de diciembre, de medidas de protección integral contra la violencia de género, propone el sistema educativo como contexto esencial para eliminar cualquier forma de violencia contra las mujeres. Asimismo, la Ley Orgánica 3/2007, de 22 de marzo, para la igualdad efectiva de mujeres y hombres, establece que desde la educación superior se fomentará la enseñanza y la investigación sobre el significado y alcance de la igualdad entre mujeres y hombres.

Por otra parte, la Ley Orgánica 4/2007, de 12 de abril, de Universidades, señala que esta Institución debe respetar la igualdad entre mujeres y hombres y crear programas específicos sobre igualdad de género. En este sentido, el Real Decreto 1393/2007, de 29 de octubre, por el que se establece la ordenación de las enseñanzas universitarias oficiales, reconoce "el respeto a los derechos fundamentales y de igualdad entre hombres y mujeres" como uno de los principios inspiradores de los planes de estudios de cualquier actividad profesional.

A nivel local, la Universidade da Coruña (UDC, 2019) aprobó el II Plan de Igualdad de la UDC con el objetivo de formar a la comunidad universitaria en varios ejes:

- Eje 1: Gobernanza, Responsabilidad Social y Transversalidad.

- Eje 2: Perspectiva de género en la actividad docente e investigadora.

- Eje 3: Prevención e Intervención frente a la violencia machista.

- Eje 4: Conciliación, desarrollo personal y carrera profesional.

Sin duda, este Plan refleja el compromiso de la UDC con la aplicación real y efectiva del principio de igualdad. No obstante, queda mucho camino por delante, pero la educación constituye una herramienta poderosa de transformación social... 


\section{DESCRIPCIÓN DE LA EXPERIENCIA}

El Grado en Terapia Ocupacional por la UDC se implanta en el curso 2008/2009. La Federación Mundial de Terapeutas Ocupacionales (WFOT, 2012) define la Terapia Ocupacional como:

"Una profesión de la salud centrada en el cliente que se ocupa de promover la salud y el bienestar a través de la ocupación. El objetivo principal de la terapia ocupacional es capacitar a las personas para participar en las actividades de la vida diaria. Los/as terapeutas ocupacionales logran este resultado trabajando con las personas y las comunidades para e as comunidades para mejorar su capacidad de involucrarse en las ocupaciones que desean, necesitan o se espera que hagan, o modificando la ocupación o el entorno para apoyar mejor su compromiso ocupacional" (World Federation of Occupational Therapy [WFOT], 2012, p.6).

El objetivo principal del Título es capacitar al alumnado para "promocionar la salud" de las personas, grupos o poblaciones con las que colabora. Para ello, es preciso abordar en el currículo, entre otros aspectos, el género, como determinante social de la salud, y la perspectiva de género.

De manera explícita y ante el contexto descrito en los antecedentes, en el curso 2012/2013 se pone en marcha en la materia optativa Terapia Ocupacional, Diversidad Funcional y Calidad de Vida, un Módulo sobre Igualdad, Género, Diversidad y Salud, destinado al alumnado de cuarto curso de la Titulación. Este módulo es el centro de la propuesta que se plantea para explicitar y visibilizar distintas temáticas cuyo eje central el género y diversas experiencias vinculadas con buenas prácticas y con vulneración de derechos en este ámbito (ver Figura 15). 


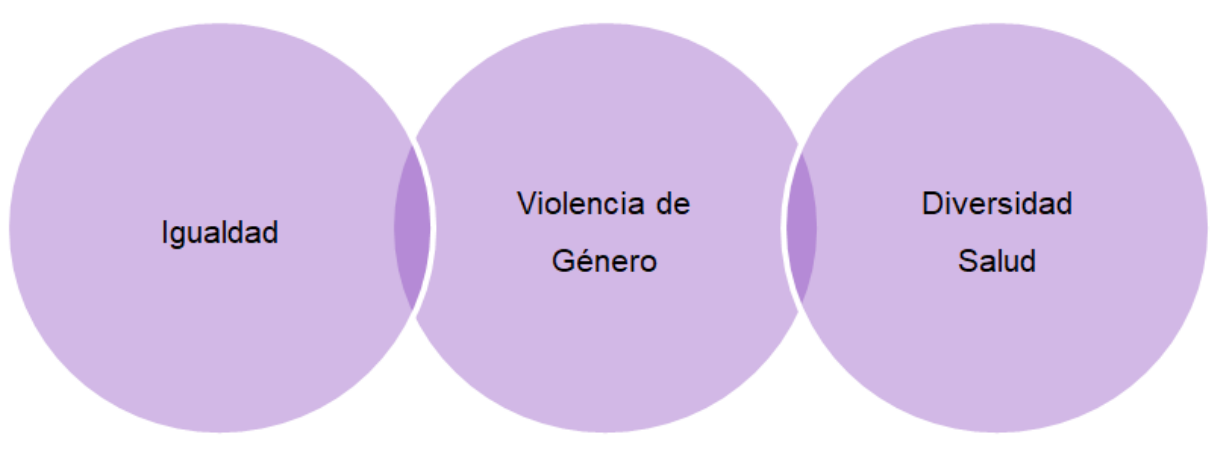

Figura 15. Ejes temáticos centrales del Módulo sobre Igualdad, Género, Diversidad y Salud

Los objetivos concretos de este módulo son:

- Desarrollar la capacidad del alumnado para evaluar las desigualdades por razón de sexo/género.

- Identificar y analizar las causas estructurales y efectos de la violencia de género en la salud y participación de las mujeres.

- Identificar otras formas de violencia contra la mujer.

- Desarrollar un razonamiento crítico y un compromiso social, respetando la pluralidad de realidades sociales.

- Saber identificar la interseccionalidad de la desigualdad de género con otros ejes de desigualdad (edad, sexualidad, identidad/expresión de género, diversidad funcional, etc.).

En esta programación se han desarrollado diversas actividades de formación y sensibilización del estudiantado, analizando el impacto que tiene el patriarcado, como sistema socio-político en la generación de espacios y contextos generadores de desigualdad y exclusión. Para ello, se han empleado metodologías de aprendizaje activo (grupos de discusión, talleres, visionado de vídeos, etc.) que implican una mayor participación del alumnado en su proceso de aprendizaje y que favorecen el pensamiento crítico, en coherencia con las temáticas tratadas. 
Cada curso académico se ofertan diversas propuestas de trabajo al alumnado posibilitando tratar temáticas de actualidad ligadas a cuestiones de género. Así, en el curso 2019/2020 se ha revisado el marco normativo existente en materia de género, así como otros documentos nacionales e internacionales relevantes para contextualizar y debatir sobre las propuestas de diversos grupos políticos. Asimismo, se ha analizado el lenguaje utilizado por distintos agentes sociales (medios de comunicación, profesionales de la salud, políticos/as, etc.). Además, se ha estudiado el impacto que tiene el género y la violencia de género en la salud y se ha discutido sobre otras formas de violencia contra mujeres y niñas. Finalmente se ha propuesto realizar una recensión sobre la mirada de género desde Terapia Ocupacional.

La evaluación de esta propuesta de innovación y responsabilidad social que aborda el género de forma específica constituye un tercio de la nota final de la asignatura, teniendo en cuenta especialmente el proceso de reflexión del alumnado.

\section{RESULTADOS}

A continuación, se detallan los principales resultados de la propuesta desarrollada. Desde el curso 2012-2013, 94 alumnos/as cursaron la materia Terapia Ocupacional, Diversidad Funcional y Calidad de Vida, con una participación activa en el Módulo de Igualdad, Género, Diversidad y Salud.

En el curso 2017/2018 y 2018/2019 se observa un aumento de Trabajos Fin de Grado (TFG) en Terapia Ocupacional (ver Figura 16), vinculados a las temáticas abordadas en este módulo: género y salud $(n=8)$, género e identidad $(n=1)$, violencia de género $(n=2)$ y sexualidad $(n=5)$. 


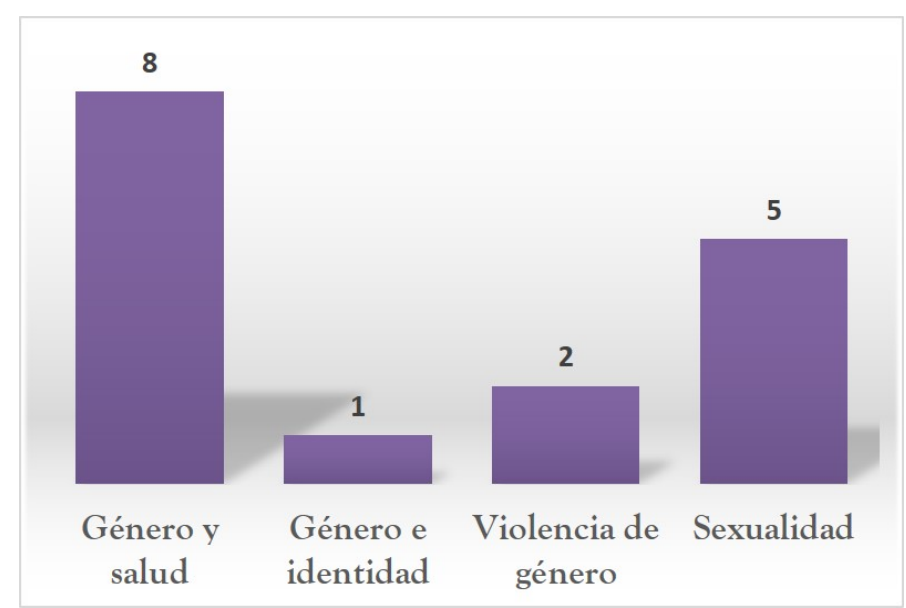

Figura 16. Resultados del número de TFG del Grado en Terapia Ocupacional relacionados con la temática de la propuesta durante el bienio 2017-2019

De igual modo, las investigaciones iniciadas en el Grado en Terapia Ocupacional impactaron en las esferas de educación y responsabilidad social. Este aspecto se refleja en diferentes acciones, como la realización de un corto, premiado por la Diputación de A Coruña, basado en un TFG; la matriculación en estudios de Posgrado relacionados con los ámbitos mencionados; la continuación de líneas de investigación iniciadas como TFG en Trabajos Fin de Máster (TFM). Asimismo, cabe destacar la elevada participación del estudiantado en actividades desarrolladas por el Centro (Facultade de Ciencias da Saúde) en materia de Igualdad e incluso la creación una plataforma feminista por parte del estudiantado de la Titulación.

\section{CONCLUSIONES}

La incorporación de la perspectiva de género en el Plan de Estudios de Terapia Ocupacional supone un aspecto que mejora la relevancia social del conocimiento y desarrolla en el alumnado una mayor sensibilidad y sentido crítico.

Los resultados muestran un creciente interés del estudiantado por cuestiones de género, así como una mayor capacitación para desarrollar investigaciones o proyectos desde esta perspectiva. 
No obstante, aplicar una mirada de género en la docencia implica una mayor formación del profesorado y pone de manifiesto la necesidad de reflexionar de forma conjunta sobre los contenidos y competencias de la Titulación de forma transversal y de las asignaturas de forma específica.

Es preciso tomar conciencia del enorme camino que tenemos por delante y del relevante papel que desempeña la Universidad como Institución transformadora y creadora de una sociedad más tolerante e igualitaria.

\section{REFERENCIAS}

Alonso, A. \& Lombardo, E. (2016). Ending ghettoization? Mainstreaming gender in Spanish political science education, European Political Science, 15 (3): 292-302.

Amorós, C. (1995). 10 palabras clave sobre mujer. Pamplona, España: Verbo Divino.

Agència per a la Qualitat del Sistema Universitari de Catalunya (2019). Marco general para la incorporación de la perspectiva de género en la docencia universitaria. Barcelona, España: AQU Catalunya

Beckwith, K. (2005). A Common Language of Gender, Politics and Gender, 1 (1): 128-137.

Cagigas, A. (2000). "El patriarcado, como origen de la violencia doméstica." Monte Buciero, 5, 307-318.

Comisión Europea (2016). Guidance on Gender Equality in Horizon 2020. European Commission - Directorate-General for Research \& Innovation.

Facio, A., \& Fries, L. (2005). Feminismo, género y patriarcado. Revista Sobre Enseñanza Del Derecho de Buenos Aires, 3(6), 259-294. https://doi.org/issn 1667-4154

Ley Orgánica 1/2004 de Medidas de Protección Integral contra la Violencia de Género. Boletín Oficial del Estado, 28 de diciembre de 2004, num 313, pp. 42166 a 42197.

Ley 3/2007, de 22 de marzo, para la igualdad efectiva de mujeres y hombres. Boletín Oficial del Estado, 23 de marzo de 2007, núm. 71, pp. 12611 a 12645. 
Organización Mundial de la Salud. (2009). Subsanar las desigualdades en una generación: alcanzar la equidad sanitaria actuando sobre los determinantes sociales de la salud. Buenos Aires, Argentina: Ediciones Journal S.A.

Real Decreto 1393/2007, de 29 de octubre, por el que se establece la ordenación de las enseñanzas universitarias oficiales. Boletín Oficial del Estado, 31 de octubre de 2007, núm. 260, pp. 44037 a 44048.

Scott, JW. (2008). Género e historia. México DF: Fondo de Cultura Económica.

Universidade da Coruña. (2019). II Plan de Igualdade 2019 - 2022. A Coruña, España Universidade da Coruña. Disponible en:

https://www.udc.es/export/sites/udc/oficinaigualdade/_galeria_down/documentos/IIPLAN-UDC_2019_2022.pdf_2063069294.pdf

World Federation of Occupational Therapists. (2012). Definitions of Occupational Therapy from member organisations, revised May 2018 [World Federation of Occupational Therapists Official Website]. Recuperado el 15 de enero de 2015, de https://wfot.org/resources/articles-of-association 